\title{
From Idea Crowdsourcing to Managing User Knowledge
}

\section{Risto Rajala, Mika Westerlund, Mervi Vuori, and Jukka-Pekka Hares}

\author{
"Crowdsourcing will shift its focus from individuals") \\ solving individual problems to a more collaboration- \\ based model. Groups of people will be engaged to solve \\ more complex problems, and the power of the \\ crowdsourcing engine will be used to create crack \\ virtual teams that you can build locally.
}

\author{
Chris McNamara \\ COO, DesignCrowd \\ tinyurl.com/k7gorac
}

\begin{abstract}
This article explores how technology companies can benefit from user knowledge in product and service innovation beyond mere idea generation through crowdsourcing. We investigate a case from the telecommunications sector to discover the ways a company can overcome the challenges of motivating users to participate in innovation activity and gaining from their knowledge in the innovation process. In particular, we seek to learn how the company has created understanding about the future uses of technology and the developments of the market with the lead users. In addition, we analyze the key means of capturing value from the knowledge gathered from the users, including the essential organizational practices that support user innovation and the ways the company makes sense of the vast volume and variety of user knowledge. Our empirical inquiry increases the understanding of how technology companies can complement and use crowdsourcing to effectively utilize knowledge resident in user communities.
\end{abstract}

\section{Introduction}

There is a growing appreciation for the value of resources that lie beyond a firm's organizational boundaries and can be tapped into for innovation purposes and R\&D collaboration with suppliers, universities, customers, or even competitors (Un et al., 2010; tinyurl.com/ mlcbg5t). Users can be considered as one important source of innovation, and user innovation has been recognized as one central research stream within the open innovation phenomenon (Gassman, 2006; tinyurl.com/n5fq3gs). The unique knowledge held by users is perceived as a valuable resource for innovation because it improves understanding of real-life situations where the company's product or service is used (Poetz and Schreier, 2012; tinyurl.com/lgham7n). Previous research shows that innovations created by lead users have been regarded commercially attractive. Moreover, it has been shown that the needs of lead users indicate how the market is to change in the future (von Hippel, 2005; tinyurl.com/57xp5x). Also, Piller and Walcher (2006; tinyurl.com/m9nkb4r) show that innovations developed with lead users can be successfully commercialized. Hence, it is reasonable to think that, from an innovation management perspective, companies should engage users - especially lead users - in ideation processes to devise desirable solutions.

Given that the knowledge needed for innovation is becoming increasingly distributed across organizational boundaries (Swan et al., 1999; tinyurl.com/cgy3gje), the task of capturing user ideas and transforming them into commercialized innovations poses a challenge for companies. Although many companies have resorted to 


\title{
From Idea Crowdsourcing to Managing User Knowledge
}

\author{
Risto Rajala, Mika Westerlund, Mervi Vuori, and Jukka-Pekka Hares
}

user design toolkits to capture users' ideas (Thomke and von Hippel, 2002; tinyurl.com/l6vb5gq), crowdsourcing has become an increasingly popular tool for acquiring external knowledge and ideas (Djelassi and Decoopman, 2013: tinyurl.com/lqfbrxg; Feller et al., 2012: tinyurl.com/l8oxsle). Crowdsourcing is characterized by the voluntary participation of a diverse crowd in a problem-solving initiative from a sponsoring organization that chooses from among the generated ideas and solutions (cf. Estellés-Arolas and Gonzales-Ladron-deGuerva, 2012; tinyurl.com/ma8ohjg). A company that initiates a crowdsourcing initiative is usually exploring innovative solutions that may include new sources of revenue in the form of new products, new services, or even new business models (Dahlander and Gann, 2010: tinyurl.com/chacrs9; Djelassi and Decoopman, 2013: tinyurl.com/lqfbrxg).

But does crowdsourcing lead to increased or improved innovation? Leimeister and colleagues (2009; tinyurl.com/adzjqv6) argue that idea contests promoting the competitive nature of idea crowdsourcing may actually lead to less collaboration and information sharing among contributors. Likewise, the absence of discourse - the ability to share various perspectives and build on each other's knowledge amongst crowdsourcing participants - can inhibit co-creation in innovation (Majchrzak and Malhotra, 2013; tinyurl.com/ mu6ypck). Although these challenges relate to the incentives associated with the implementation of crowdsourcing, the issue of how a company can actually transform knowledge generated by crowdsourcing into viable innovations that outperform the competition remains a major challenge for any organization. Thus, there is a need for more research on the mechanisms, concepts, and tools to manage the wisdom of crowds, as well as on filling the conceptual gap between the generation and the selection of ideas and their transformation into innovations (Ebner et al., 2009; tinyurl.com/mwm2yfm).

This study aims to increase the understanding on how technology companies can move beyond using crowdsourcing to collect ideas to a more systematic and nuanced way of using crowdsourcing to manage user knowledge. In particular, the study focuses on the ways an organization can utilize crowdsourcing to gather knowledge from the users and subsequently complement and use this knowledge in new product and service development. In doing so, the study examines: i) which motivations companies perceive as essential for users to share their knowledge for innovation pur- poses, ii) what the key organizational practices are that support effective user innovation management, and iii) what the key challenges are from a knowledge management perspective. We believe that addressing these questions through an empirical inquiry is of interest to scholars and practitioners of innovation.

\section{The New Role of Users as Innovators}

External contributors are becoming ever more important sources of knowledge and innovation for commercial product and service development. The literature on innovation management links customers to the success of product and service innovation (e.g., Von Hippel et al., 2011: tinyurl.com/cc98mlb; Coviello and Joseph, 2012: tinyurl.com/lkuu2qj) and suggests that users constitute a great potential source of innovation, because the competence and experience of users is not limited to the early idea generation: they can contribute throughout the innovation development process (Edvarsson et al., 2012; tinyurl.com/mvv2jbw). Through user innovation, companies can find new ideas more rapidly and at a lower cost than through traditional internal innovation. However, profiting from user innovation is difficult (Bogers et al., 2010; tinyurl.com/nxdeyb6) because user knowledge is considered complex and challenging to manage effectively.

Prior research has viewed users in different ways. An early work by Eason (1987; tinyurl.com/m4s5ewb) classifies users into three categories: i) primary users: those likely to be frequent users of the product or service; ii) secondary users: those who use the product or service through an intermediary; and iii) tertiary users: those affected by the introduction of the product or service or who will influence its purchase. Later works (e.g., Sharp et al., 2007; tinyurl.com/kpqdbot) have defined users as those who interact directly with the product to achieve a task. However, companies must not only understand the interactions of users with their products; it is also important to understand non-user behaviour, such as the reasons behind a customer's intentional decision not to take on a product or service. Also, it is important to understand the situation of people who are not yet users to possibly help them benefit from the value of use. Indeed, several scholars have stressed the importance of mobilizing a mix of users in the innovation activity. For instance, Surowiecki (2005; tinyurl.com/ ld49904) suggests that diversity among members of the crowd, independent thought on the part of the actors, and decentralization in the organization of the activity are keys to success in crowdsourcing. 


\title{
From Idea Crowdsourcing to Managing User Knowledge
}

\author{
Risto Rajala, Mika Westerlund, Mervi Vuori, and Jukka-Pekka Hares
}

Von Hippel (1986; tinyurl.com/kxznqq3) underscores that lead users take part in successful innovation. Congruently, the study of Coviello and Joseph (2012; tinyurl.com/lkuu2qj) highlights that successful user innovation often engages lead users; they are keen to participate in the innovation activity because there is potential value created for their own needs in the innovation process. In addition to engaging lead users, Coviello and Joseph (2012; tinyurl.com/lkuu2qj) suggest that those users that are technically eager, open to learning, and willing to commit to the nascent innovation are equally relevant. They show that tech-savvy users seem to be willing to learn during the innovation process and, thus, are capable to adapt to changes and provide new ideas and relevant feedback in changing situations. Moreover, Edvarsson and colleagues (2012; tinyurl.com/mvv2jbw) demonstrate the potential for experienced users to provide context-specific expertise to the innovation process.

\section{Crowdsourcing as a Form of User Innovation}

Various community-engineering techniques leverage the potential of crowds by fostering an online user community for innovation, which provides a major opportunity for R\&D (Ebner et al., 2009; tinyurl.com/mwm2yfm). Consequently, many approaches have been used to interact with users for innovation, including living labs and crowdsourcing. Companies use a variety of techniques to maximize returns from their interactions with users, and each approach has its strengths and weaknesses. In general, posting business problems in large communities - for example through "challenge driven innovation" (Bingham and Spradlin, 2011; tinyurl.com/ kw7yey9) - may expose sensitive information and strategic intent to a wide audience, but crowdsourcing offers a possibility for more focused user innovation. Pisano and Verganti (2008; tinyurl.com/luw84un) suggest that, in company-led innovation approaches, innovating with a small number of contributors is appropriate when:

- one knows the knowledge domain from which the best solution to the problem is likely to emerge

- having the best experts is important and one has the capability to pick them

- one can define the problem and evaluate the proposed solutions
Conversely, Pisano and Verganti (2008; tinyurl.com/ luw84un) suggest that a larger community of innovators may prove beneficial when:

- one requires ideas from many parties and the best ideas may come from unexpected sources

- participating in the network is easy

- the problem is small or, if large, can be broken into modular parts

- one can evaluate many proposed solutions cheaply

In its pure form, crowdsourcing is a manifestation of the latter approach. According to Pisano and Verganti (2008; tinyurl.com/luw84un), such an approach may be applicable in situations where a company is able to present a problem, anyone can propose solutions, and the company wishes to choose the solutions it likes best. However, large communities imply remarkable challenges for managing user knowledge. Knowledge in online user communities is characterized by mobility, appropriability, and stability that need to be orchestrated to make benefit of crowdsourcing (Feller et al., 2012; tinyurl.com/l8oxsle). Gibbert, Leibold, and Probst (2002; tinyurl.com/mbryalo) point out that the major challenges in making use of the knowledge resident in user communities include understanding and supporting users' motivations to participate in collaboration with a commercially oriented company. Community members' social orientations typically depart from the host organization's commercial focus, which can lead to unresolved tensions and to the failure of the initiative (Kelleher et al., 2011; tinyurl.com/ld8fecy).

In addition, users' knowledge and experiences are often tacit by nature and therefore difficult to share (Bonner, 2010; tinyurl.com/lddau6n). Users may find it challenging to share their knowledge in a meaningful way to support innovation. Moreover, Smith and McKeen (2005; tinyurl.com/kfxv927) show that structural challenges in the innovator's organization may hinder user participation. On the other hand, Jeppesen and Molin (2003; tinyurl.com/k2h6o4r) argue that user innovation can be structured, motivated, and organized by a company that provides the infrastructure for user participation. To this end, Boudreau and Lakhani (2009; tinyurl.com/ khrzmnl) argue that executives need to consider whether users are motivated to participate by intrinsic motives such as enjoyment, status, and identity that participants can gain through their interactions with others (Deci et al., 1999; tinyurl.com/k6zambt) or by extrinsic 


\title{
From Idea Crowdsourcing to Managing User Knowledge
}

\author{
Risto Rajala, Mika Westerlund, Mervi Vuori, and Jukka-Pekka Hares
}

motives such as financial benefits. In all, these notions on benefiting from online user communities call for more research on capturing, managing, and utilizing user knowledge for new product and service development.

\section{Methodology}

Our study follows the research design of an explorative single-case study where data collection took place using interviews. Extant literature on user innovation and crowdsourcing were used to guide the study; they provided us with an initial understanding of managing external innovation and users' roles in the innovation process. The inductive phases were conducted using an interpretive case study method (Walsham, 1995; tinyurl.com/nyca4vj), including seven semi-structured interviews with innovation and user community managers in the case organization (Table 1). In the interviews, the managers of the case organization were asked to share their views regarding the methods, knowledge gained, and the outcomes of crowdsourcing with their user communities. Given that we intend to improve the understanding of how the case company

Table 1. List of interviews with the managers of the case organization

\begin{tabular}{cllc}
\hline Interview & Title & $\begin{array}{l}\text { Area of } \\
\text { Responsibility }\end{array}$ & $\begin{array}{l}\text { Years at the } \\
\text { Organization }\end{array}$ \\
\hline 1 & Manager & $\begin{array}{l}\text { Manager of Beta } \\
\text { Labs }\end{array}$ & 7 \\
\hline 2 & $\begin{array}{l}\text { Senior } \\
\text { Manager }\end{array}$ & $\begin{array}{l}\text { Innovation and } \\
\text { benchmarking }\end{array}$ & 7 \\
\hline 3 & $\begin{array}{l}\text { Senior } \\
\text { Manager }\end{array}$ & $\begin{array}{l}\text { Strategy planning } \\
\text { and dialogue }\end{array}$ & 2 \\
\hline 4 & $\begin{array}{l}\text { Senior } \\
\text { Manager }\end{array}$ & $\begin{array}{l}\text { Customer culture } \\
\text { and innovation }\end{array}$ & 9 \\
\hline 5 & Trend & $\begin{array}{l}\text { Opportunity } \\
\text { identification }\end{array}$ & 10 \\
\hline 6 & Specialist & 7 \\
\hline 7 & Manager & $\begin{array}{l}\text { Service creation } \\
\text { product quality } \\
\text { and delivery }\end{array}$ & 5 \\
\hline & Manager & $\begin{array}{l}\text { Customer insight } \\
\text { initiative }\end{array}$ & \\
\hline & & & \\
\hline
\end{tabular}

may benefit from the knowledge gained through crowdsourcing, the managers involved in the crowdsourcing initiatives within the case company were considered feasible informants. The interpretations and meanings given to the different subjects by the interviewees were taken into consideration in our analysis of the data as suggested by Denzin and Lincoln (2011; tinyurl.com/ levjb4g). In addition to the interviews, we also had access to a variety of secondary data, including company reports, white papers, articles, and studies.

The company investigated in our single case study is a globally operating manufacturer of mobile phones and related devices and software. In 2010, the company employed 60,000 people from 115 different nationalities. We selected this company because it has reportedly shown interest in benefiting from their customers' knowledge in service innovation. The case company has applied crowdsourcing to make use of the skills and creativity of the users in its product and service innovation activity. It has established a separate business unit to manage user insight in its innovation activity. This unit conducts crowdsourcing projects among other user-centered innovation activities. The case provides us with an opportunity to analyze the factors that facilitate large-scale user-knowledge management through crowdsourcing. What is more, it reveals some of the lessons to be learned from the challenges of transforming crowdsourcing initiatives away from idea generation to mastering knowledge gained from the users.

We provide illustrative excerpts from the interviews to demonstrate the key findings. After transcribing the interviews, we coded the contents and organized the data to discrete yet connected blocks that describe the key themes discovered from the data. Initially, we identified four general themes in user knowledge management: i) users' motivations for knowledge sharing, ii) diversity of the participating users, iii) facilitators of user innovation, and iv) challenges in deriving business value from user knowledge. That is, the analysis revealed those motives that companies perceive as essential to support to enhance users' knowledge sharing for innovation. Moreover, the differences between the types of users surfaced in the analysis and emphasized the importance of focusing on the lead users. Finally, the analysis separates the practices that foster user innovation through crowdsourcing and the challenges faced by companies in deriving business value from users' knowledge. 


\title{
From Idea Crowdsourcing to Managing User Knowledge
}

\author{
Risto Rajala, Mika Westerlund, Mervi Vuori, and Jukka-Pekka Hares
}

\section{Theme 1: Users' Motivations for Knowledge Sharing}

\section{Product giveaways}

The willingness of users to participate in knowledge sharing and developing products and services is not connected with financial incentives. Instead, the users participate because they are interested in the products themselves: "[We] have not given [direct] monetary compensations to individual lead users, but we may have rewarded them with a [rather small] promotional product gifts (such as a phone, headset, or something like that)" (Interview 3). Monetary compensation is not among the important motivators they use to support knowledge sharing, because active participants want to be the first ones who see and get to use the new products. In addition, our interviewees underscored that users desire better and newer products and are willing to learn something new. Hence, small tangible rewards, such as the company's latest mobile devices, were seen to motivate users more than other rewards. For example, enthusiastic users submitted more than 2,500 new ideas related to mobile phones over a five-week period, just for the chance to win one of 15 new devices given away in the contest. Documents from the case show that small tangible rewards, such as the latest mobile devices, motivate people more than any other reward (tinyurl.com/k952yjs).

\section{Meritocracy}

The lead users are seen to be motivated to contribute to knowledge sharing, product development, and collaboration with a technology company in order to gain peerto-peer recognition, for example, in the voting of usergenerated ideas within the community: "The feeling of bonding with the community and possibility to influence are significant motivators" (Interview 1). The opportunity to participate and share their own thoughts and ideas was found to be an essential user incentive that company managers support. Moreover, the feeling of being part of the user community is considered an important motivator for users to share their knowledge in the user community. In addition, gaining credit, acknowledgement, and support from others in the user community were found to be effective motivators.

\section{Credibility and trust}

Users seem to be motivated to participate in the development of products that have a strong brand image. A good corporate reputation helps recruit voluntary users to cooperate with the company. Moreover, strong brands are seen to enhance the users' motivation to share their ideas and knowledge, because users can feel they are being given an exclusive opportunity to influence the products of a recognized brand: "The credibility of our brand is so strong that a bank under our corporate brand could be easily established, assuming that the bank would be a culmination of a very high level of trust" (Interview 3). One of the interviewees underscored that most of the community users she had been in contact with wanted to cooperate with the company and take part in its innovation process because they loved the brand. However, she noted that it was difficult to identify the lead users: "Seeking the lead users is harder than head hunting -- there are even firms specialized in finding lead users from blogospheres and elsewhere on the web" (Interview 3). Corporate credibility and brand image were considered to influence even the non-users given that some of the users of other brands have been willing to participate in the case company's innovation activity.

\section{Theme 2: Diversity of the Participating Users}

\section{The role of lead users}

Lead users are the primary target of user innovation in our case organization. The interviewed managers stressed that lead users are also most willing to participate in projects with the company: "The target group needs to be clear and feedback should not be collected randomly from random people" (Interview 2). The interviewees highlighted that lead users are not only enthusiastic about collaborating with the company, but they are also very interested in the latest technology and eagerly seek emerging programs because they want to try everything new. The lead users are highly capable in using the products and they have a good insight into the products: "They seem to know more about the products than what we do" (Interview 1). Users' ideas about the potential use of products go far beyond technological thinking about the future evolution of the products. Because lead users bring out novel ways to use the product in the future, it is important to understand the character and living context of the lead users: "pure ideas are not important, the people behind them are" (Interview 3).

\section{User needs reflecting future trends}

Lead users' perceptions were deemed important in the case organization because they are considered to represent the future needs of the mainstream users. However, sometimes the needs of lead users are so advanced that their behaviours never become mainstream. The preferences of lead users and the mainstream may differ significantly; some features that lead users may rate highly may be of no interest to the 


\title{
From Idea Crowdsourcing to Managing User Knowledge
}

\author{
Risto Rajala, Mika Westerlund, Mervi Vuori, and Jukka-Pekka Hares
}

average user. Companies must take this into account when working with lead users. Crowdsourcing can significantly benefit from a mix of users given that it aims to collect a variety of ideas and knowledge. However, "lead users are the ones who most often volunteer to participate in the projects with the company" (Interview 3) and "it is more difficult to reach the mainstream" (Interview 6). Thus, it is tempting to focus on lead users that form their own homogenous community; they share ideas and thoughts with each other and want to be members in communities with like-minded others having similar interests. They are opinion leaders about technology and are considered to not only affect the innovation, but also the social behaviour of their friends and peers.

\section{Theme 3: Facilitators of User Innovation}

\section{Mechanisms of participation}

Our interviewees underscored the importance of paying attention to the ways of participating, gathering, and processing ideas. In addition to crowdsourcing, the case company has used various methods to collect customer knowledge, including workshops, interviews, ethnography, anthropology, consumer feedback, online events, forums, blogs, communities, focus groups, consumer testing, tracking, quantitative methods, open source, design reviews, and surveys. Furthermore, they use toolkits for involving consumers in the development process: "We have invited lead users to the brainstorming events. They come there of their own accord and we pay the expenses, and of course we're trying to make it a 'wow'-experience" (Interview 3). Users share their experiences, and the company tries to capture an impression of their everyday lives. One of the informants found that this is a way to identify important details, which the users may not even be conscious of or perceive as important. Therefore, it is important for an observer to have an analytical eye for the tacit knowledge embedded in the practices of everyday life.

\section{Selection of relevant knowledge}

Recognizing and picking relevant information is a major concern in large-scale crowdsourcing: "How do we obtain the right knowledge, and on which level should the relevant user information be brought in so that it matches the needs of our in-house innovation? We can understand the world but we cannot control its needs so to say, because they are emerging and changing all the time. That is a big problem" (Interview 5). It is also not always clear which part of the user input should be taken seriously. As disclosed by one of our informants (Interview 3), people may overstate their expertise in or- der to become chosen into the crowdsourcing program. Another consideration is that the participants may represent only a small fraction of the users and that the most enthusiastic users may be overrepresented. "Some people like our brand so much they participate in these events eagerly" (Interview 3). The company was seen as the leader of the process of recognizing and deciding the needs behind the users' behaviour, because the users do not usually care about the expenses or how large a customer segment their idea would serve: "We must be the brains that decide what customers need; we cannot assume they tell themselves about the needs the customer is not even aware of yet" (Interview 4). Yet, the interviewees emphasized the importance of being able to put oneself in the user's shoes: "You need to have a correct mindset all the time; you need to have a user in mind. Moreover, you need to use different sources of information and then decide and pick the relevant points. It's more a matter of competence than matter of the volume of information" (Interview 4).

\section{Continuity of interaction}

The analysis shows that, in order to gain long-haul innovation outcomes, collaboration with the users should run on a continual basis. Conversely, the knowledge should be used promptly by the company. The interviewees all felt that the crowdsourcing processes must be kept simple and straightforward: "The process should not go like this: you first plan a study and then order it and then get it sometime in the future. No way, that would be too slow" (Interview 2). The innovation development process should be as quick as possible and users' ideas should be utilized soon after capturing them. The process of collecting feedback should be continuous so that the company has the newest ideas available all the time. That way, the whole process becomes closer to a partnership and makes the best use of crowdsourcing. Users should be engaged in the innovation process throughout the product lifecycle.

\section{Theme 4: Challenges in Deriving Business Value from User Knowledge}

\section{Contingencies of knowledge}

The tacit nature of knowledge poses major challenges to making use of users' knowledge. Tacit knowledge is probably the most challenging to collect due to its ambiguity and implicit characteristics. Tacit knowledge gathered from users can be best utilized when obtained in person. One of the interviewees said that, in her business unit, user knowledge is exploited effectively because they are doing ethnography research where the knowledge is gained mostly by personal involvement: 


\title{
From Idea Crowdsourcing to Managing User Knowledge
}

\author{
Risto Rajala, Mika Westerlund, Mervi Vuori, and Jukka-Pekka Hares
}

"I am not sure to what degree this kind of tacit knowledge is exploited in other firms at the moment" (Interview 5). The tacit nature of knowledge was seen as one reason why data repositories are not a feasible solution from the effective-utilization perspective. Tacit knowledge was considered to be best gained in face-to-face interaction and all of the interviewees mentioned that the only ways to collect tacit knowledge are personal interaction and working with users and observing them in action.

\section{Sharing the acquired knowledge internally}

To derive business value from the user-induced knowledge, the organization should be capable of utilizing the knowledge in its innovation process: "There are people who want to collaborate with us to develop our devices and services and they have many ideas, but we need a system to make use of their input." (Interview 2). The company's internal knowledge-sharing practices and cross-functional integration were perceived important in effective utilization of external knowledge.

\section{Making sense of the data gained}

The case company has conducted a large-scale project to make sense of all the knowledge obtained from the users. The sensemaking activities include data visualization where the outcome is a two-dimensional "idea map" (tinyurl.com/k952yjs). The visualization is based on advanced text-mining combined with clustering and regression analysis (Vuori, 2012; tinyurl.com/lbn3c2c). Through the idea map, a company can, for example, spot weak signals and megatrends: "The visualizations of user-generated ideas on a map allow us to concentrate on the most relevant knowledge. For the organization's strategy people and $R \& D$ specialists, the visualized map of user knowledge is a refined view of the continuously evolving ideas and contributions from users." (Interview 7). The idea map also contributes to deepening the understanding of the lifecycle of a certain segment. Such an understanding supports decisions regarding the technology roadmap.

\section{Discussion}

The findings discussed above provide a rationale to suggest that capturing and making use of knowledge resident in online user communities comprises four interlinked processes: management of community, management of ideas, management of innovation, and management of knowledge. Furthermore, management of information exchange between these processes is crucial, because the company assigns tasks and design challenges to the crowd and then reaps the rewards of their contributions to the processes. Whereas crowdsourcing is an effective method to promote and collect user ideas in large communities, our findings suggest that there is a need to proceed from mere collection of ideas through crowdsourcing to management of user knowledge. To capture the value of user-induced knowledge, researchers and practitioners should consider the following key takeaways of this study:

1. Users value easy sharing of their knowledge for user innovation. There are several methods available to collect knowledge from users, including workshops, interviews, crowdsourcing, netnography, living labbing, web analytics, and online market research techniques. In addition, there are a myriad of channels for gathering user input, such as idea competitions, and different ways to organize online events and focus groups, observation of user communities, consumer testing, tracking, design reviews, opinion polls, and toolkits for involving users in the development process. Those channels that have best fit with individuals' behaviour are the most effective regarding quality, credibility, and relevance of the knowledge gathered.

2. Continuous interaction with the lead users and acknowledging the users for their ideas are vital in effective user innovation. The findings highlighted that continuous interaction between the firm and its user community is crucial for innovation, and collected ideas should be assessed and implemented quickly. Furthermore, our findings show that gaining tacit knowledge from the users requires profound collaboration with the users. Therefore, we suggest that users should be engaged in the innovation process throughout the whole product lifecycle, or for a prolonged period instead of through separate encounters.

3. Good internal knowledge management practices are important. Critical processing of the acquired knowledge is vital. In practice, the experiences of the company underlined that unitary data repositories fail to make a viable solution to user knowledge management, as they cannot scale to large volumes of data. Moreover, the variety and velocity of user knowledge is often immense and cannot be standardized. According to our findings, the tacit nature of knowledge is a reason for the major challenges of user-knowledge management practices in crowdsourcing. Hence, it calls for advanced data analytics capabilities. 


\title{
From Idea Crowdsourcing to Managing User Knowledge
}

\author{
Risto Rajala, Mika Westerlund, Mervi Vuori, and Jukka-Pekka Hares
}

4. Making sense of the data gained is a key to creating value with user knowledge. Data visualization is one of the key activities pursued by our case company in its effort to make sense of the areas of knowledge and in the practical aim of sharing the relevant knowledge with those intra-firm actors that need it most. This activity has proven to be one of the keys to create value with the ideas and knowledge gained from the users. The case company has made a great use of data mining and clustering techniques to provide both the strategy process and individual R\&D projects with relevant ideas to support their specific needs out of the bunch of data collected.

\section{Conclusion}

How do the findings improve our understanding of using crowdsourcing in online user communities to source user knowledge for innovation? Although crowdsourcing is an effective way to collect ideas from large communities of heterogeneous users, our study shows that companies need to think about user-knowledge management in a more holistic way to complement and make benefit of users' knowledge. Furthermore, the study suggested four key lessons to move beyond mere idea crowdsourcing. First, technology companies need to understand and support users' motives for knowledge sharing. Although users are willing to share their ideas for free, effective incentives include the opportunity to gain access to the latest products or services, and the possibility of receiving token gifts as a reward. Second, given that user-knowledge management is often time-consuming and requires considerable effort, companies should pay attention to choosing the right users for collaboration. The case organization valued lead users, but recognized their potential bias in representing average users. Third, companies need to implement processes and practices that support user innovation and knowledge sharing. Companies can improve their innovation performance by sharing user knowledge in social action between those actors participating in the innovation process instead of collecting all data in one repository. Fourth, companies need to focus on how to visualize the data and make sense of the relevant information when using large-scale user ideation methods such as crowdsourcing in order to derive business value from users' knowledge.

\section{Acknowledgments}

The authors wish to express their gratitude to the $\mathrm{Fu}$ ture Industrial Services (FutIS) research program. Also, the support of the Finnish Funding Agency for Technology and Innovation (Tekes) and the Finnish Metals and Engineering Competence Cluster (FIMECC) is gratefully acknowledged.

\section{About the Authors}

Risto Rajala, D.Sc. (Econ) is an Assistant Professor in the Department of Industrial Engineering and Management at Aalto University in Helsinki, Finland. Dr. Rajala holds a PhD in Information Systems Science from the Aalto University School of Business. His recent research has dealt with management of complex service systems, development of digital services, service innovation, and business model performance. Rajala's specialties include management of industrial services, collaborative service innovation, knowledge management, and design of digital services.

Mika Westerlund, D. Sc. (Econ) is an Assistant Professor at Carleton University's Sprott School of Business in Ottawa, Canada. He previously held positions as a Postdoctoral Scholar in the Haas School of Business at the University of California Berkeley and in the School of Economics at Aalto University. Mika earned his doctoral degree in Marketing from the Helsinki School of Economics. His doctoral research focused on software firms' business models and his current research interests include open and user innovation, business strategy, and management models in high-tech and serviceintensive industries.

\section{Continued on next page...}




\title{
From Idea Crowdsourcing to Managing User Knowledge
}

Risto Rajala, Mika Westerlund, Mervi Vuori, and Jukka-Pekka Hares

\begin{abstract}
About the Authors (continued)
Mervi Vuori, M. Sc. (Econ) is a researcher and doctoral candidate at Department of Industrial Engineering and Management at Aalto University in Helsinki, Finland. Since 2010, she has acted as a principal researcher in several research projects in the field of purchasing and innovation management. She is currently working on her doctoral dissertation on "Innovating and collaborating with external resources: crowds, communities and suppliers". Her research is centered on the use of external resources, related management interfaces, as well as integration mechanisms in service and business model innovation.
\end{abstract}

Jukka-Pekka Hares, M. Sc. (Econ) received his master's degree from the Aalto University School of Economics in Helsinki, Finland. His master's thesis focused on crowdsourcing and user knowledge management in online user communities. He is currently working at the public relations agency Manifesto as a communications consultant.

Citation: Rajala, R., M. Westerlund, M. Vuori, and J.-P.

Hares. 2013. From Idea Crowdsourcing to Managing

User Knowledge. Technology Innovation Management

Review. December 2013: 23-31.

Keywords: crowdsourcing, user innovation, online

communities, knowledge management, lead users 